\title{
A LINEAR GENERALIZATION OF GRONWALL'S INEQUALITY
}

\author{
D. WILLETT
}

1. In the study of ordinary differential equations and integral equations, there often occurs inequalities of the form

$$
u(t) \leqq w_{0}(t)+\int_{0}^{t} k(t, s) u(s) d s \quad(t>0),
$$

where $k(t, s)$ and $w_{0}(t)$ are known non-negative functions and $u(t)$ is an unknown non-negative function. For examples, one can refer to Bellman [1, pp. $35 \mathrm{ff}$.$] , Coddington and Levinson [2, pp. 37 \mathrm{ff}$.$] ,$ Willett [3], and others. In order to obtain from (1.1) a genuine upper bound for $u(t)$, i.e., an upper bound independent of $u$, it seems necessary to separate the variable $t$ in $k(t, s)$ from the integrand involving $u(s)$. This can be done by assuming that $k(t, s)$ is directly separable, i.e., that there exist measurable functions $v_{i}(t)$ and $w_{i}(t)(i=1,2, \cdots, n)$ such that

$$
k(t, s) \leqq \sum_{i=1}^{n} w_{i}(t) v_{i}(s),
$$

or by applying Hölder's inequality to $\int_{0}^{t} k(t, s) u(s) d s$. The latter is the so-called $L_{P}$ case and is analyzed in [3].

In $\$ 2$ of this note we state as Theorem 0 the case when $k(t, s)$ is directly separable with $n=1$. A special case of this result was first published by Gronwall [4], and a result of nearly the same generality of Theorem 0 appears in [2, p. 37]. The purpose of this note is to prove theorems for general $n$ when either $k(t, s)$ or $\partial k(t, s) / \partial t$ is directly separable. Actually we need only that some derivative of $k(t, s)$ with respect to $t$ be directly separable, but there seems to be no need to produce the details beyond the first derivative case since the procedure is clear.

An example illustrating the usefulness of the results for $n>1$ is given in $\$ 3$.

2. In what follows all functions are assumed to be real-valued and defined on a given interval $I$ with zero as left endpoint. The domain of $k(t, s)$ is taken to be the subset of $I \times I$ for which $t \geqq s$. All functions are assumed Lebesgue measurable and all functions of one variable are

Received by the editors June 8, 1964. 
assumed non-negative. We call such a function $x=x(t)$ locally integrable on $I$ if for each $t \in I$, its Lebesgue integral $\int_{0}^{t} x(s) d s=\int_{0}^{t} x$ is finite.

THEOREM 0. Suppose that

$$
u(t) \leqq w_{*}(t)+w(t) \int_{0}^{t} v(s) u(s) d s \quad(t \in I),
$$

where $v w, v w_{*}$, and $v u$ are locally integrable on $I$. Then

$$
u(t) \leqq w_{*}(t)+w(t)\left(\exp \int_{0}^{t} v w\right)\left(\int_{0}^{t} v w_{*}\right) \quad(t \in I) .
$$

For the proof of Theorem 0 , one can refer to [3] or [2, p. 37]. We state Theorem 0 here in order to simplify the proofs of the following two theorems:

Theorem 1. Suppose that

$$
u(t) \leqq w_{0}(t)+\sum_{i=1}^{n} w_{i}(t) \int_{0}^{t} v_{i}(s) u(s) d s \quad(t \in I),
$$

where $v_{i} w_{j}(i=1,2, \cdots, n ; j=0,1, \cdots, n)$ and $v_{i} u(i=1,2, \cdots, n)$ are locally integrable on $I$. Then

$$
u \leqq E_{n} w_{0},
$$

where $E_{i}(i=0,1, \cdots, n)$ is defined inductively as the composition of $i+1$ functional operators, i.e., $E_{i}=D_{i} D_{i-1} \cdots D_{0}$, where

$$
\begin{aligned}
& D_{0} w=w \\
& D_{j} w=w+\left(E_{j-1} w_{j}\right)\left(\exp \int_{0}^{t} v_{j} E_{j-1} w_{j}\right) \int_{0}^{t} v_{j} w \quad(j=1,2, \cdots, n) .
\end{aligned}
$$

Proof. For $n=1$ the theorem reduces to Theorem 0 , and hence is true. Suppose $n$ is given and $n>1$. The proof is by finite induction. Assume the following two statements (A) and (B) hold for $i=k$, where $k$ is some integer between 0 and $n-1(0 \leqq k \leqq n-1)$ :

(A) $E_{i} w_{j}$ exists and $v_{m} E_{i} w_{j}$ is locally integrable

$$
(j=i+1, i+2, \cdots, n, 0 ; m=i+1, i+2, \cdots, n) ;
$$

(B) $u \leqq E_{i} w_{0}+\sum_{m=i+1}^{n}\left(E_{i} w_{m}\right) \int_{0}^{t} v_{m} u$. 
(A) and (B) hold by assumption for $i=k=0$, and we will prove that (A) and (B) hold for $i=k+1$ if $k \leqq n-2$ and that the theorem is true if $k=n-1$. In either case, the theorem follows.

Let $w=E_{k} w_{j}$. From the definition of $E_{k+1}$, we obtain

$$
E_{k+1} w_{j}=D_{k+1} w=w+\left(E_{k} w_{k+1}\right)\left(\exp \int_{0}^{t} v_{k+1} E_{k} w_{k+1}\right) \int_{0}^{t} v_{k+1} w .
$$

It follows from (A) with $i=k$ and the local integrability of $v_{k+1} w_{0}$ that $E_{k+1} w_{j}(j=k+1, k+2, \cdots, n, 0)$ exists and that $v_{m} E_{k+1} w_{j}(m=k+1$, $k+2, \cdots, n ; j=k+1, k+2, \cdots, n, 0)$ is locally integrable. Hence, (A) holds for $i=k+1$, and we may apply Theorem 0 with $v=v_{k+1}$ to (B) with $i=k$ to obtain

$$
u \leqq w_{0}^{*}+\left(E_{k} w_{k+1}\right)\left(\exp \int_{0}^{t} v_{k+1} E_{k} w_{k+1}\right) \int_{0}^{t} v_{k+1} w_{0}^{*},
$$

where

$$
\begin{aligned}
w_{0}^{*} & =E_{k} w_{0}+\sum_{m=i+2}^{n}\left(E_{k} w_{m}\right) \int_{0}^{t} v_{m} u & & \text { if } k \leqq n-2, \\
& =E_{k} w_{0} & & \text { if } k=n-1 .
\end{aligned}
$$

Substituting from (2.8) for $w_{0}^{*}$ in (2.7), rearranging the terms, and using the fact that $\int_{0}^{t} v_{m} u$ is a nondecreasing function of $t$, we can in the case $k \leqq n-2$ obtain from (2.7) inequality (B) with $i=k+1$. If $k=n-1$, we can get from (2.7) equation (2.3), which is the conclusion of the theorem.

In the next theorem we will show how an inequality of the same form as (2.3) can be produced in the case that $k(t, s)$ is differentiable and $\partial k(t, s) / \partial t$ rather than $k(t, s)$ is directly separable.

Theorem 2. Suppose that inequality (1.1) holds and that $\partial k(t, s) / \partial t$ exists in the domain of $k(t, s)$ and there

$$
\frac{\partial k}{\partial t}(t, s) \leqq \sum_{i=1}^{n} w_{i}(t) v_{i}(s)
$$

Let $r(t)$ be a non-negative measurable function such that

$$
k(t, t) \leqq r(t)
$$

(We are assuming still that all functions of one variable are non-negative.) If $v_{i} u$ and $w_{i}(i=1,2, \cdots, n), r$, and $r w_{0}$ are locally integrable on $I$, then 


$$
u(t) \leqq w_{0}^{*}(t)+\sum_{i=1}^{n} w_{i}^{*}(t) \int_{0}^{t} v_{i}(s) u(s) d s
$$

where

$$
\begin{aligned}
& w_{0}^{*}(t)=w_{0}(t)+\int_{0}^{t} r(s) w_{0}(s)\left(\exp \int_{0}^{t} r\right) d s, \\
& w_{i}^{*}(t)=\int_{0}^{t} w_{i}(s)\left(\exp \int_{s}^{t} r\right) d s \quad(i=1,2, \cdots, n) .
\end{aligned}
$$

Proof. Define a differentiable function $\theta(t)$ on $I$ by

$$
\theta(t)=\int_{0}^{t} k(t, s) u(s) d s .
$$

Compute the derivative $\theta^{\prime}(t)$ and then use equation (1.1) to obtain

$$
\theta^{\prime}(t) \leqq r(t) w_{0}(t)+r(t) \theta(t)+\int_{0}^{t} \frac{\partial k}{\partial t}(t, s) u(s) d s .
$$

Next, transpose $r(t) \theta(t)$ and multiply the inequality by $\exp \left(-\int_{0}^{t} r\right)$ to produce

$$
\begin{aligned}
& \left(\theta \exp \left(-\int_{0}^{t} r\right)\right)^{\prime} \\
& \quad \leqq r w_{0} \exp \left(-\int_{0}^{t} r\right)+\left(\exp \left(-\int_{0}^{t} r\right)\right) \int_{0}^{t} \frac{\partial k}{\partial t}(t, s) u(s) d s .
\end{aligned}
$$

Now substitute for $\partial k(t, s) / \partial t$ from (2.9) and integrate between zero and an arbitrary point of $I$. Inequality (2.10) follows from the monotonicity of $\int_{0}^{t} v_{i} u(i=1,2, \cdots, n)$ by substituting for $\theta(t)$ in (1.1).

Inequality (2.10) is of the same form as inequality (2.3); hence, if the integrability assumptions of Theorem 1 are satisfied in this situation, Theorem 1 can be applied to produce a bound on $u(t)$.

3. As an example illustrating the greater usefulness of Theorems 1 and 2 relative to Theorem 0 , consider the inequality

$$
u(t) \leqq t+\lambda^{2} t \int_{0}^{t} e^{-\lambda s} u(s) d s+\int_{0}^{t} u(s) d s, \quad(t>0) .
$$

Here $\lambda$ is a real parameter and the problem is to determine the asymptotic behaviour of $u$ as $\lambda \rightarrow+\infty$, in particular, to prove that $u=O(1)$ uniformly for $t$ restricted to compact subintervals of $[0,+\infty)$. We have 


$$
k(t, s)=\lambda^{2} t e^{-\lambda_{e}}+1 ;
$$

hence, $\partial k(t, s) / \partial t=\lambda^{2} e^{-\lambda \ell}$. The application of Theorem 2 is straightforward and leads to the desired result. We could have also put $v_{1}(t)$ $=w_{1}(t)=1, w_{2}(t)=t$, and $v_{2}(t)=\lambda^{2} e^{-\lambda t}$ and successfully applied Theorem 1 . On the other hand, the direct application of Theorem 0 in the obvious fashion,

$$
k(t, s) \leqq \max \left(1, \lambda^{2} t\right) e^{-\lambda}
$$

or

$$
k(t, s) \leqq\left(1+\lambda^{2} t\right) \max \left(1, e^{-\lambda s}\right),
$$

does not produce that $u$ must be bounded as $\lambda \rightarrow+\infty$.

\section{REFERENCES}

1. R. Bellman, Stability theory of differential equations, McGraw-Hill, New York, 1953.

2. E. Coddington and N. Levinson, Theory of ordinary differential equations, McGraw-Hill, New York, 1955.

3. D. Willett, Nonlinear vector integral equations as contraction mappings, Arch. Rational Mech. Anal. 15 (1964), 79-86.

4. T. H. Gronwall, Note on the derivatives with respect to a parameter of the solutions of a system of differential equations, Ann. of Math. 20 (1918), 292-296.

University of Alberta, Edmonton, Alberta, Canada 